\title{
Breaking AKR1C3-mediated enzalutamide resistance by inhibiting androgen synthesis
}

Upregulation of $A K R 1 C 3$ expression and resultant intracrine androgens in prostate cancer cells is a mechanism of resistance to enzalutamide that can be overcome by the NSAID indometacin, suggests a new study by Chengfei Liu and colleagues from the University of California at Davis, Sacramento, USA.

Androgen synthesis by tumour cells is one of the processes leading to development of castration-resistant prostate cancer (CRPC). Although treatment with the androgen-receptortargeting drug enzalutamide can provide a survival benefit in men with CRPC, tumour cells frequently develop resistance to this agent. The interplay between intratumoral androgen expression and enzalutamide resistance is an area of intensive research. "Understanding the potential mechanisms driving enzalutamide resistance in CRPC will aid in the

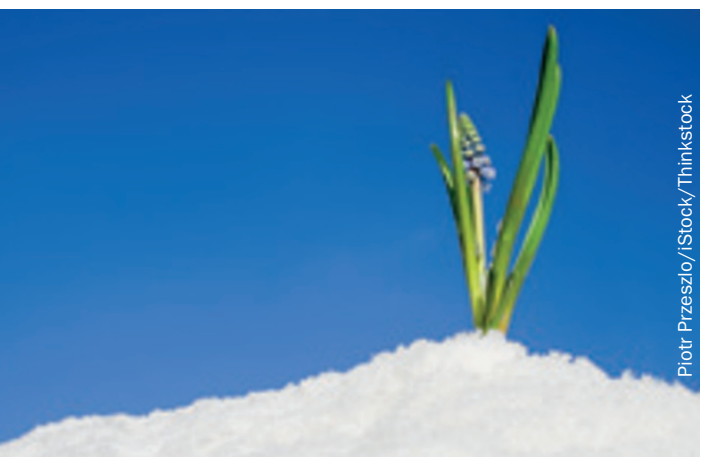

development of next-generation treatments that target these mechanisms," explains Allen Gao, senior author of the paper.

\section{4 ...inhibition of $A K R 1 C 3$ pathways could act as an enzalutamide-sensitizing treatment... 77}

In their study, the researchers first identified variations in the expression of genes involved in steroid hormone biosynthesis in a previously generated enzalutamide-resistant cell line (C4-2B MDVR) using microarray analysis. Of the 31 genes that showed upregulation, protein expression was increased for three genes (AKR1C3, HSD3B and CYP17A1) compared with the nonresistant parental cell line. The team also found increased levels of $17-\beta$-HSD 5 , the enzyme expressed from $A K R 1 C 3$, in three other enzalutamide-resistant cell lines and in samples of tumour xenografts. Evaluation of AKR1C3 expression profiles of normal and malignant prostate tissues stored in online databases demonstrated that the enzyme, which catalyzes the last step in testosterone biosynthesis, is highly expressed in late-stage prostate cancer.

Next, Liu and colleagues demonstrated that their resistant C4-2B MDVR cells express very high levels of testosterone, dihydrotestosterone and dehydroepiandrosterone, as well as some androgen precursors. Importantly, when suppressing 17- $\beta$-HSD 5 expression using short hairpin RNAs in C4-2B MDVR cells and another enzalutamide-resistant cell line or stably expressing $17-\beta$-HSD 5 in LNCaP cells, the cells regained or lost sensitivity to enzalutamide treatment, respectively.

Finally, treatment of resistant cells with indometacin, which has previously been shown to inhibit 17- $\beta$ HSD 5 activity, significantly inhibited tumour cell growth in combination with enzalutamide treatment in vitro. In vivo, indometacin alone significantly suppressed tumour growth, an effect that was further enhanced in combination with enzalutamide treatment.

The data suggest that inhibition of AKR1C3 pathways could act as an enzalutamide-sensitizing treatment and restore efficacy in patients with enzalutamide-resistant CRPC. "We are moving ahead to validate our findings on $A K R 1 C 3$ and intracrine androgens in patients with advanced prostate cancer and are planning clinical trials to test targeting AKR1C3 pathways to improve enzalutamide therapy," Gao concludes.

Clemens Thoma

Original article Liu, C. et al. Intracrine androgens and AKR1C3 activation confer resistance to enzalutamide in prostate cancer. Cancer Res. doi:10.1158/ 0008-5472.CAN-14-3080 